\title{
EXISTENCE AND UNIQUENESS OF SOLUTIONS FOR A NONLINEAR SECOND ORDER DIFFERENTIAL EQUATION IN HILBERT SPACE
}

\author{
by DANG DINH HAI
}

(Received 14th June 1988)

This paper is concerned with the existence and uniqueness of solutions for the Picard boundary value problem

$$
x^{\prime \prime}(t)+k x^{\prime}(t)+f\left(t, x(t), x^{\prime}(t)\right)=0, \quad x(0)=x(\pi)=0
$$

in a real Hilbert space. Our theorems improve corresponding results of Mawhin for $|k|$ large.

1980 Mathematics subject classifications (1985 Revision): 34G20, 34B15.

\section{Introduction}

Let $H$ be a real Hilbert space. We consider the following Picard boundary value problem in $H$

$$
\begin{gathered}
x^{\prime \prime}(t)+k x^{\prime}(t)+f\left(t, x(t), x^{\prime}(t)\right)=0, \quad t \in I \\
x(0)=x(\pi)=0
\end{gathered}
$$

where $I=[0, \pi], f: I \times H \times H \rightarrow H$ and $k \in \mathbb{R}$.

The problem (1)-(2) was studied in [2] for the case $H=\mathbb{R}^{n}$, where references to the corresponding literature are also given. The results in [2] were generalized to the case of a Hilbert space by Mawhin in [3]. The purpose of this note is to establish some existence and uniqueness results, which extend (but do not contain) the corresponding results of Mawhin [3]. Our approach is based on the Leray-Schauder fixed point theorem.

\section{Existence and uniqueness theorems}

We first set some notations.

We denote by $(\cdot, \cdot)$ the inner product in $H$ and by $|\cdot|$ the corresponding norm. The norm in $C(I, H), C^{1}(I, H)$ and $L^{2}(I, H)$ will be denoted by $|\cdot|_{0},|\cdot|_{1}$ and $\|\cdot\|$ respectively.

Theorem 1. Suppose that: 
(i) $f: I \times H \times H \rightarrow H$ is completely continuous;

(ii) $k \neq 0$ and there exist nonnegative numbers $a, b, c$ with

$$
a+\frac{b^{2}}{4}<\frac{|k|}{2 \pi\left(1-e^{-|k| \pi}\right)}
$$

such that

$$
(x, f(t, x, y)) \leqq a|x|^{2}+b|x||y|+c|x|
$$

for all $t \in I$ and all $x, y \in H$;

(iii) there exist a continuous function $h: \mathbb{R}^{+} \rightarrow \mathbb{R}^{+}$and a constant $K$ such that

$$
\int_{M / \pi}^{K} \frac{d s}{h(s)+|k|} \geqq 2 M
$$

where

$$
\begin{gathered}
M=\left(2 a+b^{2}\right) \pi R^{2}+2 \pi c R \\
R=\frac{2 \pi c\left(1-e^{-|k| \pi}\right)}{|k|-2 \pi\left(a+b^{2} / 4\right)\left(1-e^{-|k| \pi}\right)}
\end{gathered}
$$

and

$$
|(y, f(t, x, y))| \leqq h\left(|y|^{2}\right)|y|^{2}
$$

for all $t \in I, y \in H$ and $x \in H$ such that $|x| \leqq R$. Then the problem (1)-(2) has at least one solution.

Proof. Define the operator $A: C^{1}(I, H) \rightarrow C^{1}(I, H)$ by

$$
A x(t)=-\frac{1-e^{-k t}}{e^{k \pi}-1} \int_{0}^{\pi} e^{k s}\left(\int_{s}^{\pi} N x(\tau) d \tau\right) d s+e^{-k t} \int_{0}^{t} e^{k s}\left(\int_{s}^{\pi} N x(\tau) d \tau\right) d s
$$

where $N x(\tau)=f\left(\tau, x(\tau), x^{\prime}(\tau)\right)$.

It is easy to see that $A$ is completely continuous and that the problem (1)-(2) is equivalent to the fixed point problem $x=A x$. To apply the Leray-Shauder fixed point theorem, we look for a constant $C$ such that for all possible solutions of the equations

$$
x(t)=\lambda A x(t), \quad t \in I, \quad \lambda \in(0,1)
$$

or, cquivalently, 


$$
x^{\prime \prime}(t)+k x^{\prime}(t)+\lambda N x(t)=0, \quad x(0)=x(\pi)=0
$$

we have

$$
|x|_{1}<C
$$

Now let $x$ be a possible solution of (11) with $\lambda \in(0,1)$. Then

$$
\left(x^{\prime \prime}(\tau), x(\tau)\right)+k\left(x^{\prime}(\tau), x(\tau)\right)+\lambda(N(x(\tau), x(\tau))=0
$$

i.e.,

$$
\left(x^{\prime}, x\right)^{\prime}(\tau)-\left|x^{\prime}(\tau)\right|^{2}+(k / 2)\left(|x|^{2}\right)^{\prime}(\tau)+\lambda(N x(\tau), x(\tau))=0
$$

Integrating (12) over $(s, \pi)$ and using the boundary conditions, we get

$$
-2\left(x^{\prime}(s), x(s)\right)-k|x(s)|^{2}+2 \int_{s}^{\pi}\left[\lambda(N x(\tau), x(\tau))-\left|x^{\prime}(\tau)\right|^{2}\right] d \tau=0
$$

or, after multiplication of both members by $e^{k s}$,

$$
-\left(e^{k s}|x(s)|^{2}\right)^{\prime}+2 e^{k s} \int_{s}^{\pi}\left[\lambda\left(N(x(\tau), x(\tau))-\left|x^{\prime}(\tau)\right|^{2}\right] d \tau=0\right.
$$

Integrating (13) over $(0, t)$ and using the boundary conditions, we get

$$
|x(t)|^{2}=2 e^{-k t} \int_{0}^{t} e^{k s}\left(\int_{s}^{\pi}\left[\lambda\left(N(x(\tau), x(\tau))-\left|x^{\prime}(\tau)\right|^{2}\right] d \tau\right) d s\right.
$$

We claim that

where $R$ is defined by (7).

$$
|x|_{0} \leqq R
$$

Indeed, by (4) and Cauchy's inequality,

$$
(N x(\tau), x(\tau)) \leqq\left(a+b^{2} / 4\right)|x(\tau)|^{2}+c|x(\tau)|+\left|x^{\prime}(\tau)\right|^{2} .
$$

Assume first that $k>0$. By (14) and (16),

$$
|x(t)|^{2} \leqq 2 \pi \frac{1-e^{-k t}}{k}\left[\left(a+b^{2} / 4\right)|x|_{0}^{2}+c|x|_{0}\right], \quad t \in I
$$


from which (15) follows.

Suppose next that $k<0$. By rewriting (14) as

$$
|x(t)|^{2}=2 e^{-k t} \int_{t}^{\pi} e^{k s}\left(\int_{0}^{s}\left[\lambda\left(N(x(\tau), x(\tau))-\left|x^{\prime}(\tau)\right|^{2}\right] d \tau\right) d s\right.
$$

and using (16), we deduce

$$
|x(t)|^{2} \leqq 2 \pi \frac{1-e^{-|k|(\pi-t)}}{|k|}\left[\left(a+b^{2} / 4\right)|x|_{0}^{2}+c|x|_{0}\right], \quad t \in I
$$

from which (15) follows. This proves the claim.

Taking the inner product of (11) with $-x(t)$ and integrating over $I$ give

$$
\begin{aligned}
\left\|x^{\prime}\right\|^{2} & =\lambda \int_{0}^{\pi}\left(N(x(t), x(t)) d t \leqq \pi a|x|_{0}^{2}+\sqrt{\pi} b|x|_{0}\left\|x^{\prime}\right\|+c \pi\|x\|_{0}\right. \\
& \leqq \pi\left(a+b^{2} / 2\right)|x|_{0}^{2}+c \pi|x|_{0}+(1 / 2)\left\|x^{\prime}\right\|^{2}
\end{aligned}
$$

which implies, by (15),

$$
\left\|x^{\prime}\right\|^{2} \leqq \pi\left(2 a+b^{2}\right) R^{2}+2 \pi c R=M
$$

Hence, by the mean value theorem, there exists $t_{0} \in I$ such that

$$
\left|x^{\prime}\left(t_{0}\right)\right|^{2} \leqq M / \pi
$$

Now, taking the inner product of (11) with $x^{\prime}(t)$ gives, by (8),

$$
\left.\left.\left|\frac{d}{d t}\right| x^{\prime}(t)\right|^{2}\left|\leqq 2\left(h\left(\left|x^{\prime}(t)\right|^{2}\right)+|k|\right)\right| x^{\prime}(t)\right|^{2}
$$

or

$$
\left|\frac{d}{d t} \int_{0}^{\left|x^{\prime}(t)\right|^{2}} \frac{d s}{h(s)+|k|}\right| \leqq 2\left|x^{\prime}(t)\right|^{2}
$$

By the mean value theorem, (5) and (18)-(20), it follows that 


$$
\int_{0}^{\left|x^{\prime}(t)\right|^{2}} \frac{d s}{h(s)+|k|} \leqq \int_{0}^{M / \pi} \frac{d s}{h(s)+|k|}+2 M \leqq \int_{0}^{K} \frac{d s}{h(s)+|k|} \text { for all } t \in I .
$$

Hence

$$
\left|x^{\prime}\right|_{0} \leqq K
$$

which completes the proof of Theorem 1 .

Theorem 2. Suppose that:

(i) $f: I \times H \times H \rightarrow H$ is continuous;

(ii) $k \neq 0$ and there exist nonnegative numbers $a, b$ with

$$
a+b^{2} / 4<\frac{|k|}{2 \pi\left(1-e^{-|k| \pi}\right)}
$$

such that

$$
(x-u, f(t, x, y)-f(t, u, v)) \leqq a|x-u|^{2}+b|x-u||y-v|
$$

for all $t \in I$ and all $x, y, u, v \in H$.

Then the problem (1)-(2) has at most one solution.

Proof. Let $x, u$ be two solutions of (1)-(2). Put $z=x-u$. Then

$$
\begin{gathered}
z^{\prime \prime}(t)+k z^{\prime}(t)+f\left(t, x(t), x^{\prime}(t)\right)-f\left(t, u(t), u^{\prime}(t)\right)=0 \\
z(0)=z(\pi)=0 .
\end{gathered}
$$

As in the proof of Theorem 1, we deduce

$$
|z(t)|^{2}=2 e^{-k t} \int_{0}^{t} e^{k s}\left(\int_{s}^{\pi} p(\tau) d \tau\right) d s=2 e^{-k t} \int_{1}^{\pi} e^{k s}\left(\int_{0}^{s} p(\tau) d \tau\right) d s
$$

where

$$
p(\tau)=\left(f\left(\tau, x(\tau), x^{\prime}(\tau)\right)-f\left(\tau, u(\tau), u^{\prime}(\tau)\right), z(\tau)\right)-\left|z^{\prime}(\tau)\right|^{2}
$$

Since

$$
p(\tau) \leqq\left(a+b^{2} / 4\right)|z(\tau)|^{2} \quad \text { for all } \tau \in I
$$

it follows from (22) that 


$$
|z(t)|^{2} \leqq 0, \quad t \in I
$$

which proves Theorem 2.

Remarks. 1. Theorem 1 gives conditions under which (1)-(2) has a solution without the smallness assumption on $a$ and $b$. As is well known, such an assumption is essential in the proof of many earlier results.

2. We note that (3) is satisfied for nonnegative numbers $a, b$ verifying

$$
a+b<\frac{|k|}{2 \pi\left(1-e^{-|k| \pi}\right)} \text { and } b \leqq 4
$$

In Theorem 1 of [3], it is assumed that $h: \mathbb{R}^{+} \rightarrow \mathbb{R}^{+} \backslash\{0\}$ is continuous and $h+|k|$ satisfies the 2-Nagumo condition i.e.

$$
\int_{0}^{\infty} \frac{d s}{h(s)+|k|}=\infty
$$

Mawhin proved an existence result to (1)-(2) for completely continuous $f$ satisfying (4) with $a, b, \geqq 0, a+b<1$ and verifying (8) for all $t \in I, y \in H$ and $x \in H$ with $|x| \leqq$ $\pi(1-a-b)^{-1} c$. Thus if we assume that

$$
\frac{|k|}{1-e^{-|k| \pi}}>2 \pi
$$

and that (8) holds for all $t \in l$ and all $x, y, \in H$, then the assertion of our Theorem 1 is stronger than the one in Theorem 1 of [3]. In Theorem 2 of [3], uniqueness of a solution is established for continuous $f$ satisfying (21) with $a, b, \geqq 0$ and $a+b<1$. Thus our Theorem 2 strengthens Theorem 2 of [3] for the case where (23) holds.

3. We mention that a similar result to Theorem 1 was established in [1] for the following periodic boundary value problem in $\mathbb{R}$

$$
\begin{gathered}
x^{\prime \prime}(t)+f(x(t)) x^{\prime}(t)+g(t, x(t))=e(t), \quad t \in[0,2 \pi] \\
x(0)-x(2 \pi)=x^{\prime}(0)-x^{\prime}(2 \pi)=0 .
\end{gathered}
$$

Acknowledgements. The author wishes to thank the referee for having suggested the present simplified proof of Theorem 1.

\section{REFERENCES}

1. Dang Dinh HaI, On the existence of periodic solutions for Lienard's equation, Math. Nachr. 139 (1988), 245-249. 
2. J. Mawhin, Topological Degree Methods in Nonlinear Boundary Value Problems (Regional Conf. Series in Math. 40, Amer. Math. Soc. Providence, R.I., 1979).

3. J. Mawhin, Two point boundary value problems for nonlinear second order differential equations in Hilbert spaces, Tôhoku Math. J. 32 (1980), 225-233.

Department of Mathematics

Dal Hoc TONG Hop

HoChiMinh City University

VIETNAM 